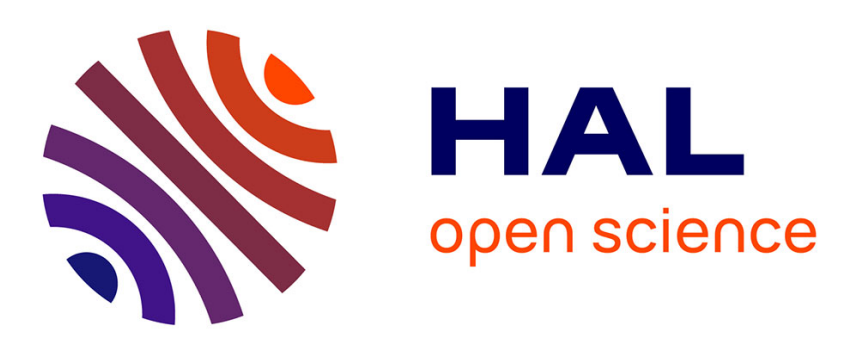

\title{
Algorithm and knowledge engineering for the TSPTW problem
}

Stefan Edelkamp, Max Gath, Tristan Cazenave, Fabien Teytaud

\section{To cite this version:}

Stefan Edelkamp, Max Gath, Tristan Cazenave, Fabien Teytaud. Algorithm and knowledge engineering for the TSPTW problem. IEEE Symposium on Computational Intelligence in Scheduling (CISched), Apr 2013, Singapour, Singapore. pp.44 - 51, 10.1109/SCIS.2013.6613251 . hal-01406484

\section{HAL Id: hal-01406484 https://hal.inria.fr/hal-01406484}

Submitted on 1 Dec 2016

HAL is a multi-disciplinary open access archive for the deposit and dissemination of scientific research documents, whether they are published or not. The documents may come from teaching and research institutions in France or abroad, or from public or private research centers.
L'archive ouverte pluridisciplinaire HAL, est destinée au dépôt et à la diffusion de documents scientifiques de niveau recherche, publiés ou non, émanant des établissements d'enseignement et de recherche français ou étrangers, des laboratoires publics ou privés. 


\title{
Knowledge and Algorithm Engineering for the TSPTW Problem
}

\author{
Stefan Edelkamp*, Max Gath*, Tristan Cazenave ${ }^{\dagger}$, and Fabien Teytaud ${ }^{\ddagger}$ \\ *Institute for Artificial Intelligence, University of Bremen, Germany, Email: \{edelkamp,mgath\}@tzi.de \\ ${ }^{\dagger}$ LAMSADE, Universite Paris Dauphine, France, Email: cazenave@lamsade.dauphine.fr \\ ‡Univ Lille Nord de France, ULCO, LISIC, BP 719, 62228 CALAIS Cedex, Email: teytaud@lisic.univ-littoral.fr
}

\begin{abstract}
In this paper we consider knowledge and algorithm engineering in combinatorial optimization for improved solving of complex TSPs with Time Windows. In addition to Nested MonteCarlo Search with Policy Adaption, as invented by Rosin (2011) and applied to TSP by Cazenave and Teytaud (2012), among other refinements to speed-up the exploration we perform beam search for an improved compromise of search breadth and depth and automated knowledge elicitation to seed the distribution for the exploration. We show promising results on TSPTW benchmarks and indicate improvements for real-world logistics scenarios by using a multiagent simulation system with each agent computing and trading their individual TSPTW solutions.
\end{abstract}

\section{INTRODUCTION}

In the Traveling Salesman Problem (TSP) a set of $N$ cities (one of which is the depot) and their pairwise distances are given. The task is to find the shortest route that starts and ends at the depot and visits each city only once.

In the Traveling Salesman Problem with Time Windows (TSPTW), additionally to the TSP, each city has to be visited and left within a given time interval. As the Hamiltonian Path problem is a subproblem, TSP, TSPTW and most other TSP variants are computationally hard [6], so that no algorithm polynomial in $N$ is to be expected.

A genetic search solver for TSPTW problems has been contributed by Potvin and Bengio [19]. Alternative algorithms are constraint logic programming [18], ant colony optimization [15], and generalized insertion heuristics [12]. Exact TSPTW solvers often apply branch-and-bound search, are usually based on refined bounds [6] and have, e.g., been suggested by [2], [7], [8], [10]. Their scaling, however, is limited.

In real-world logistics applications more general TSP(TW) variants are common: capacities limitations on the vehicle of the traveling salesman, combined delivery and backhault transportation of goods, premium vs. non-premium tasks, vehicle routing problems with several salesmen to jointly solve a logistics problem too large for one salesman, see [17] for a survey. Solomon [23] provides benchmarks for vehicle routing problems with time window constraints.

Many complex TSP problems have a huge state space and no good heuristic to order moves so as to guide the search toward the best positions, so that randomized search can be of help. For example, Nested Monte-Carlo Search uses random rollouts at its base level. It combines nested calls with randomness in the rollouts and memorization of the best tour.
Nested Monte-Carlo search has been combined with expert knowledge and an evolutionary algorithm [20]. The MonteCarlo algorithm (with a small level) is reapeatedly called and optimized by a Self-Adaptation Evolution Strategy. However, the effectiveness of this hybrid Nested Monte-Carlo algorithm decreases as the number of cities increases. Biasing MonteCarlo simulation through Rapid Action Value Estimation (RAVE) in the TSPTW domain has been investigated by Rimmel et al. [21], while Rosin [22] invented Policy Adaption for Nested Monte-Carlo search.

In this paper we perform algorithm engineering to speed-up the process of finding good TSPTW solutions. Among other implementation refinements we use beam search and policy priors. For the TSP, policy priors can be deduced from the pairwise city-to-city distance table, or from a lower bound function like the Hungarian algorithm solving the Assignment Problem (AP) or one of its refinements [13]. Moreover, we show how to elicit knowledge from the definition of the TSP to drive the solution process towards finding good solutions even more quickly.

The paper is structured as follows. We start with (Nested) Monte-Carlo Search and Policy Adaptation, and describe known domain-dependent heuristic enhancements to reduce the set of successors in the randomized search. Then, we consider code refactoring and further speed-up techniques that we jointly cast as algorithm engineering. Next, we will see that prior knowledge implemented into an initial policy can greatly reduce search efforts. The experiments are drawn on a selection of TSPTW benchmarks and show improvements to Nested Monte-Carlo search, so that more instances can be solved optimally. Finally, we conclude and discuss the adaptation of the algorithms in an industrial strength multiagent simulation system.

\section{Monte-CARlo SEARCH}

Monte-Carlo Search is a class of randomized tree search algorithms that backup values from the leaves of the search tree back to the decision nodes to direct the search towards the best solution found, while maintaining exploration breadth. Thus, the algorithm is one proposed solution to the well-known exploration vs. exploitation dilemma in state space search. 

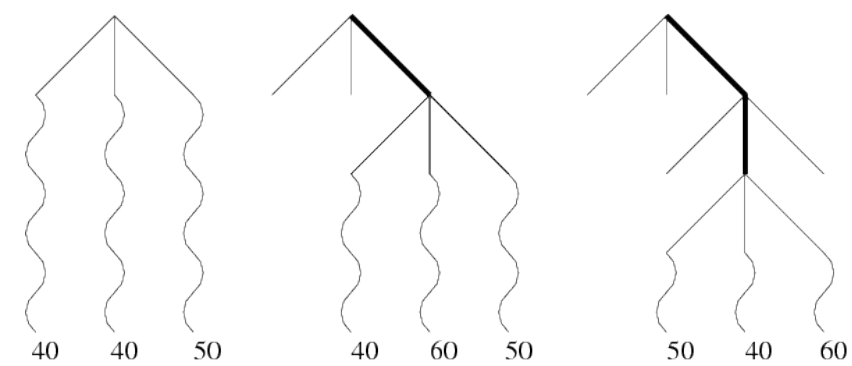

Fig. 1. This figure illustrates three successive decision steps of a level 1 search for a maximization problem. Numbers at the bottom of branches represent the rewards. Level 1 exploration is represented in thin lines. MonteCarlo playouts (i.e. level 0) are shown with wavy lines and decisions are represented with bold lines.

\section{A. UCT}

One noticeable representative in this class is the UCT algorithm [14] that dynamically builds a search tree, from whose leaves random walks to the end of the game (rollouts) are initiated. Within the explicit UCT search tree, the algorithm chains down from the root node, and applies a specialized formula that mimics a multi-armed bandit problem to select the best successor [1] of each node. The UCT-formula includes the expected payoff in form of and an exploration term, which in more recent work sometimes is dropped or substituted in favor to search knowledge in form of a value of an expert-given or otherwise learned evaluation function. UCT is very successful in playing games and outperforms traditional approaches minimax search with $\alpha \beta$ pruning, e.g., in Go [11] and General Game Playing [9], [16].

\section{B. Nested Monte-Carlo Search}

For single-agent search challenges, Nested Monte-Carlo (NMC) has been suggested and successively been applied to games like Morpion Solitaire, SameGame, Sudoku and many others [4].

NMC is a recursive algorithm that performs a certain number of rollouts, where a rollout is a random path in the search tree starting from the root and ending at a leaf that can be evaluated to some score value. The search method in NMC takes the level $l$ (initiated by $k$ ) as an argument and decrements the value by 1 in every of the number of iterations many recursives calls. If the value has decreased to 1 (or to 0 depending on the implementation), a rollout is initiated. At each choice point of a rollout the algorithm chooses the successor that gives the best score when followed by a single random rollout. Similarly, for a rollout of level $l$ it chooses the successor node that gives the best score when followed by a rollout of level $l-1$. Hence, the core objective in NMC is that the search is intensified with increasing depth of the search.

A level 1 maximization example is presented in Figure 1. The leftmost tree illustrates the start. A Monte-Carlo playout is performed for all 3 possible decisions. At the end of each playout a reward is given, and the decision with the best reward is chosen.
In [20] domain-dependent TSP heuristics have been added to bias the Monte-Carlo simulations according to a (Boltzmann softmax) policy, e.g., preferring states with a smaller distance to the last city, a smaller amount of wasted time because a city is visited too-early, or a smaller amount of time left until the end of the time window of a city.

\section{Policy Adaption in Nested Monte-Carlo Search}

The basis for our algorithm is the implementation of Cazenave and Teytaud [5] for NMC with Policy Rollout Adaption (NPRA), an algorithm originally proposed by [22]. The NMC edge learning algorithm shares similarities the RAVE adaptations applied to UCT search [21]. The rollout is thus biased on a policy $P(u, v)$ for the state space edges $(u, v)$. NPRA applies a level $l$ search with $i$ iterations in which the best policy $P_{l}(u, v)$ is updated. Moreover, in the first iteration $P_{l}(u, v)$ is initialized with $P_{l-1}(u, v)$.

\section{Domain-Dependent Pruning}

Cazenave and Teytaud [5] have enhanced NPRA with pruning rules. These extensions are domain-dependent and are based on the following two preference rules for successor set selection within the rollout.

1) If a time-window constraint is violated extending a partial tour to a successor node $v$, reduce the successor node set to $\{v\}$. The reason is that this violated city should have been visited earlier.

2) Avoid visiting a node if it makes another node fail a (time window) constraint. For this case it considers all successor nodes that do not make any other node to fail a constraint.

\section{Algorithmic Refinements}

We have performed extensive refactoring and algorithm engineering to enhance the exploration efficiency. The objective of the tuning is simple: the faster the node expansion and the rollouts implementations are, the better the nested search, as it will have more back-up information for decision making.

\section{A. Avoiding Copy Construction}

In the original implementation for TSPTW solving with NPRA by [5] the copy constructor is called in each iteration and each level of the search (thus, for each search node). This elegant solution (realized by calling copy $=\star$ this) helps understanding the difference of recursive invocation of the search and reinitializing it in each of the iterations. Moreover, parallelization of the search is made easy, as each constructor call can be given to a different computing node. However, for this case of copy construction all non-static member variables of the search class are replicated and copied to the new NMC search class instance. 


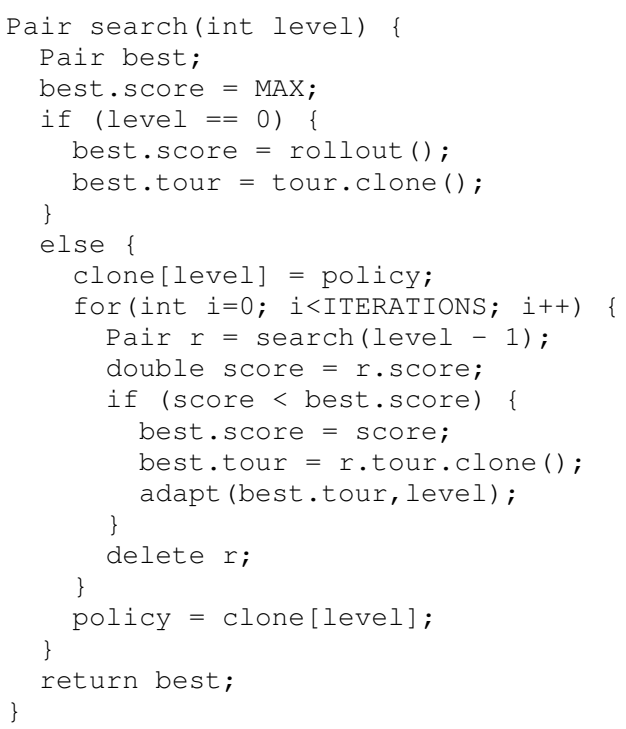

Fig. 2. Nested Monte-Carlo with Policy Adaption.

\section{B. Reducing Memory Allocation}

At each search node our implementation (see Fig. 2) copies the policy when going down from level $l-1$ to level $l$. To avoid dynamic memory allocation all policies $P_{l}, l=k, \ldots, 0$, in a Level- $k$ search are pre-allocated. Moreover, we moved the copying of the policy to a working temporary and back from inside the iteration loop to its outside.

At each search node, memory for one tour is reserved and deleted in case no better solution has been found. Hence, the memory consumption of the refined algorithm is bounded by $O\left(k \cdot N^{2}\right)$. The time efforts at each node are bounded by $O\left(N^{2}\right)$. This includes the efforts for policy adaption in case of an established improved solutions. Since successor generation takes $O\left(N^{2}\right)$ steps (due to the second heuristics) and given that it is applied to each node in the tour to be generated, for each rollout $O\left(N^{3}\right)$ operations are required.

\section{Merging Rollout and Evaluation}

In our engineered implementation we avoided the replay of the partial tour in the successor generation function for determining its makespan. Thus, we merged successor generation with the rollout. Moreover, we integrated the evaluation of a tour to a score value into the rollout procedure (see Figure 3). The offset penalizes constraint violations and is set to the predefined maximum value for the distances divided by the number of cities $N$ (This is the largest possible values if MAX is used as an upper bound for the worst possible score. (In related research $10^{6}$ is taken.)

\section{Varying Nestedness}

It is known that a Level- $k$ NMC search for a smaller value of $k$ tends to saturate earlier for a large number of node expansions than a Level- $(k+1)$ search, so that in order to find optimal solutions in bigger problem instances, larger values $k$ are often more effective. Thus, we varied $k$ and adapted the

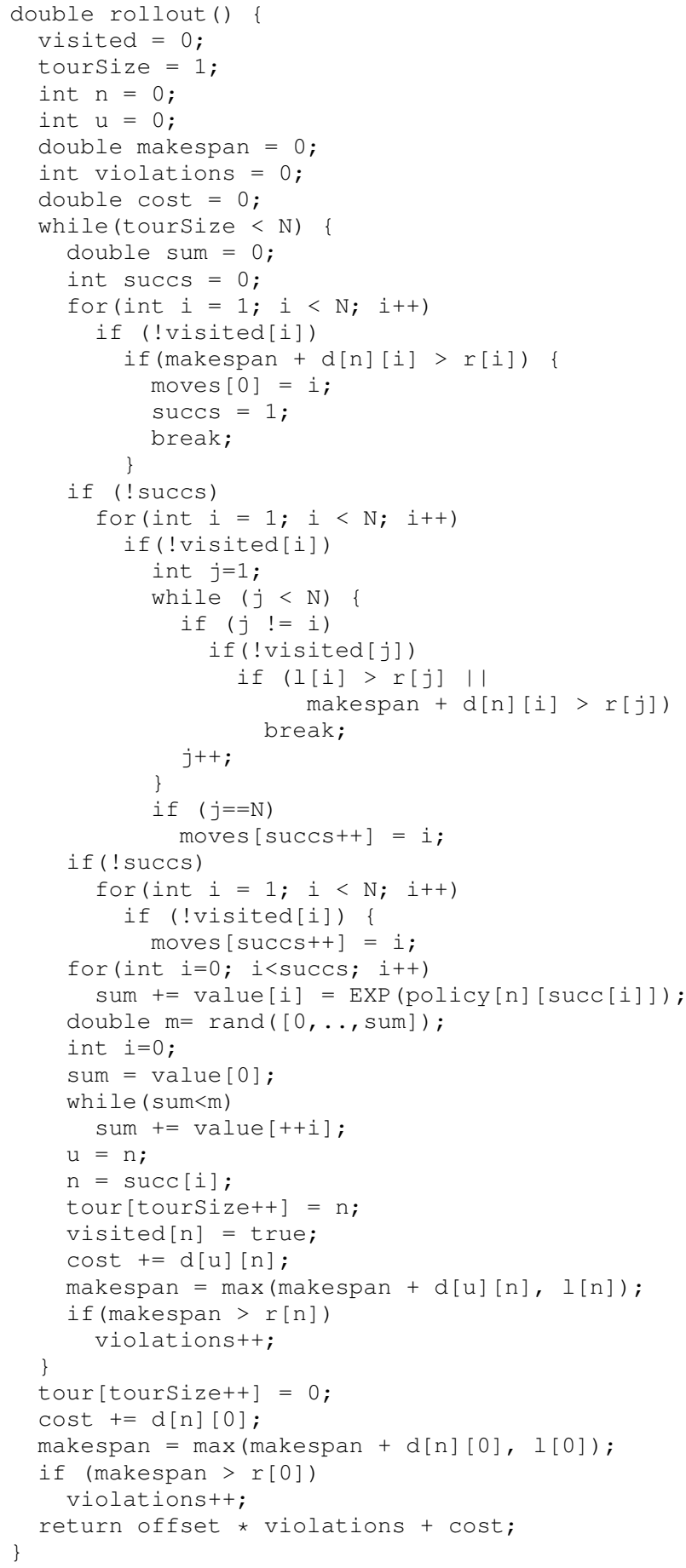

Fig. 3. Rollout with score evaluation at search tree leaf. 


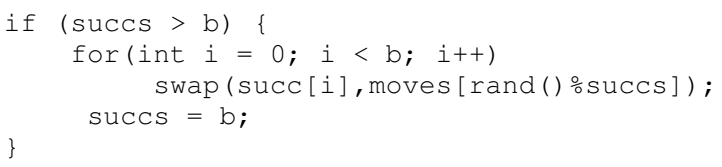

Fig. 4. Implementing beam search.

number of iterations $t$ for learning, so that between 100 million and one billion rollouts are performed for an entire exploration. As the number of rollouts is fixed $\left(t^{k}\right)$, finding an appropriate value $t$ for a given value $k$ and tree size is immediate. For example, we choose $(k, t)=(5,50)$ with a total of 312500000 rollouts (used by [5], [20]), $(k, t)=(8,12)$ with a total of 429981696 rollouts and $(k, t)=(10,7)$ with a total of 282475249 rollouts.

\section{E. Employing Beam Search}

We also experimented with Monte-Carlo beam search, as this was effective in many single-agent search domains [3]. Morpion Solitaire this enhancement helped to match the record score of 82 moves. There is, of course, a trade-off between depth and width. It is often the case that a smaller set of successors already yields a good solutions and that early failures do not harm. The smaller the number of successors the faster the rollout. Our implementation of beam search (see Fig. 4) is a simplification of Monte-Carlo beam search as recently proposed in [3]. For the experiments, we chose a beam width $b$ of $N / 2$ so that at the root node half of the successors are neglected from the search. During the rollout the relative size of the set of successors increases with the search depth.

\section{F. Adapting Knowledge}

The NMC algorithm with Policy Adaption (see Figure 5) usually is invoked with $P_{0}(u, v)=0$ for all $u, v \in\{0, \ldots N-$ $1\}$. The policy is stored in form of a $(N \times N)$-sized array and is updated the edge probabilities $P(u, v)=P_{l}(u, v)$ wrt. to an cost-improving tour as shown in the algorithm. First the denominator $z$ for normalization is computed. Then, the influence of the (chosen node / successor node) pair updates the policy.

We found that much of the time is spend in evaluating the $e$ function, so we chose known approximation for it (see Fig. 6).

\section{G. Elicitation of Knowledge}

For a probabilistic prior policy in form of an initial seed we aim at the simple strategy of including city-to-city distances. This matches the idea of reordering in depth-first branch-andbound solvers for the problem. As we want to direct the search towards successors with small distances, given that the $e$ function is applied to the policy values, we take the negative of the distance value for the policy.

In order to adjust the amplitude of these numbers, and contributing to the fact that we can exclude some edges (e.g., $(u, u)$, or $(u, v)$ with $\left.l_{u}+d_{u, v}>r_{v}\right)$ by setting their distances to infinity, we divide each value by the smallest value in

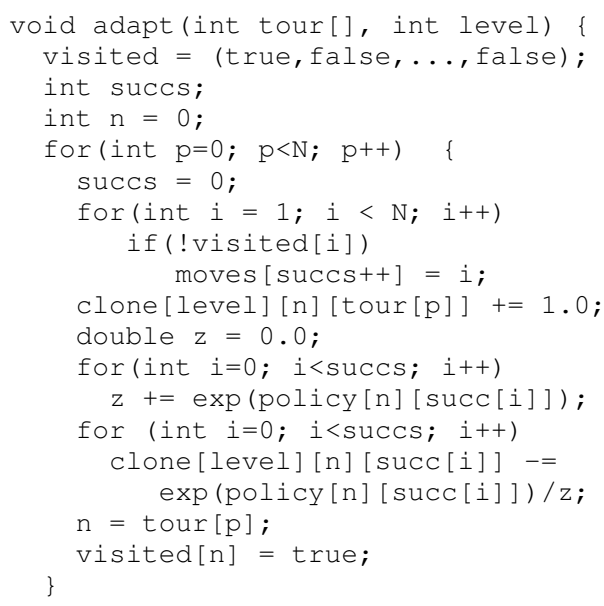

Fig. 5. Policy adaption.

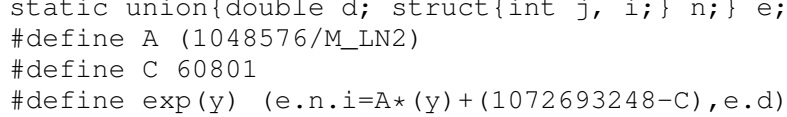

Fig. 6. Approximating the $e$-function.

one column (equivalently row) of the distance matrix. More formally, let $c_{u}=\min _{v=0}^{N-1}\left\{d_{u, v}\right\}$ be the column minima for $u \in\{0, \ldots N-1\}$. Then, we define the initial policy by $P_{0}(u, v)=-d_{u, v} / c_{u}$ for $u, v=\{0, \ldots N-1\}$.

There are other forms of knowledge available. E.g. after applying the Assignment Problem heuristic (e.g., with the cubic time Hungarian algorithm), the distance matrix is reduced to the solution of one minimal assignment. Even though for seeding the policy this lower bound has to be computed only once, we took an engineered version of its computation documented by [8].

\section{EXPERIMENTS}

We executed our experiments on one core of an Intel (R) Core (TM) i7 CPU PC at $2668 \mathrm{MHz}$ that is equipped with $8192 \mathrm{MB}$ cache and 8 GB RAM running Ubuntu Linux 11.10. We used the GNU c-compiler $g++$ (version 4.3.3), and all program compilations were optimized with -03 . For easy referencing we call our approximate TSPTW solver mTSP.

\section{A. Dumas Benchmark}

Table I shows that mTSP always finds an optimal tour for simpler benchmark instances with $N=20$. However, since the algorithm does not stop automatically, no proof certificate for optimality is derived. As expected, mTSP is much faster than the two provably optimal algorithms suggested by [8]. Since it is an anytime algorithm, the running time is set to a predefined threshold to terminate the search. We used mTSP also in larger Dumas' benchmarks with a timeout of 15 minutes (see Figure II). For $N=40$ all but 3 problems (total deviation from optimum $4+4+9=17$ ) were solved with the state-ofthe-art scores, For the $N=60$ problems all but 9 problems 
TABLE I

RESULTS IN SMALLER INSTANCES OF THE DUMAS TSPTW BENCHMARK (EXPANDED NODES $E$ / INITIATED ROLLOUTS $R$ AND CPU TIMES $T$ ARE SHOWN, INDEX $h$ AND $c$ REFER TO A DEPTH-FIRST BRANCH-AND-BOUND SOLVER WITH TWO DIFFERENT ADMISSIBLE HEURISTICS, WHILE $m$ REFERS TO MTSP, COST SHOWS THE BEST KNOWN SOLUTIONS).

\begin{tabular}{|c|r|rr|rr|rr|}
\hline Problem & Cost & $E_{h}$ & $T_{h}$ & $E_{c}$ & $T_{c}$ & $R_{m}$ & $T_{m}$ \\
\hline n20w20.001 & 378 & 49 & $<1 s$ & 2784766 & $<1 s$ & 20736 & $<1 s$ \\
n20w20.002 & 286 & 97 & $<1 s$ & 3234936 & $<1 s$ & 125000 & $<2 s$ \\
n20w20.003 & 394 & 138 & $<1 s$ & 4944477 & $<1 s$ & 125000 & $<2 s$ \\
n20w20.004 & 396 & $156<1 s$ & 2331312 & $<1 s$ & 20736 & $<1 s$ \\
n20w20.005 & 352 & $41<1 s$ & 4017260 & $<1 s$ & 125000 & $<2 s$ \\
n20w40.001 & 254 & 38022 & $3 \mathrm{~s}$ & 103087541 & $18 \mathrm{~s}$ & 125000 & $<2 s$ \\
n20w40.002 & 333 & 88 & $<1 s$ & 11388523 & $2 \mathrm{~s}$ & 20736 & $<1 s$ \\
n20w40.003 & 317 & 1409 & $<1 s$ & 21158796 & $3 \mathrm{~s}$ & 125000 & $<2 s$ \\
n20w40.004 & 388 & 7676 & $1 \mathrm{~s}$ & 35117607 & $6 \mathrm{~s}$ & 20736 & $<1 s$ \\
n20w40.005 & 288 & 10287 & $2 \mathrm{~s}$ & 20801644 & $3 \mathrm{~s}$ & 20738 & $<1 s$ \\
n20w60.001 & 335 & 40810 & $14 \mathrm{~s}$ & 223904879 & $43 \mathrm{~s}$ & 41472 & $<1 s$ \\
n20w60.002 & 244 & 97144 & $7 \mathrm{~s}$ & 81367918 & $15 \mathrm{~s}$ & 20738 & $<1 s$ \\
n20w60.003 & 352 & 399127 & $27 \mathrm{~s}$ & 31292739 & $5 \mathrm{~s}$ & 125000 & $<10 s$ \\
n20w60.004 & 280 & 4055453 & $258 \mathrm{~s}$ & 1245195466 & $238 \mathrm{~s}$ & 2509056 & $<10 s$ \\
n20w60.005 & 338 & 105393 & $10 \mathrm{~s}$ & 104049862 & $18 \mathrm{~s}$ & 2757888 & $<10 s$ \\
n20w80.001 & 329 & 316992 & $35 \mathrm{~s}$ & 288653549 & $56 \mathrm{~s}$ & 125000 & $<2 s$ \\
n20w80.002 & 338 & 260552 & $36 \mathrm{~s}$ & 166880630 & $33 \mathrm{~s}$ & 500000 & $<10 s$ \\
n20w80.003 & 320 & 15959 & $3 \mathrm{~s}$ & 208526467 & $42 \mathrm{~s}$ & 20736 & $<1 s$ \\
n20w80.004 & 304 & 1258898 & $80 \mathrm{~s}$ & 373077547 & $73 \mathrm{~s}$ & 20736 & $<1 s$ \\
n20w80.005 & 264 & 5224435 & $438 \mathrm{~s}$ & 1660621704 & $332 \mathrm{~s}$ & 125000 & $<30 s$ \\
n20w100.001 & 237 & 1635101 & $52 \mathrm{~s}$ & 1232596799 & $279 \mathrm{~s}$ & $20736 r$ & $<1 s$ \\
n20w100.002 & 222 & 68954 & $7 \mathrm{~s}$ & 2203174867 & $531 \mathrm{~s}$ & 625000 & $<30 s$ \\
n20w100.003 & 310 & 13382035 & $765 \mathrm{~s}$ & 2586795810 & $538 \mathrm{~s}$ & 20736 & $<1 s$ \\
n20w100.004 & 349 & 34289 & $2 \mathrm{~s}$ & 1213551958 & $266 \mathrm{~s}$ & 41472 & $<1 s$ \\
n20w100.005 & 258 & 688887 & $44 \mathrm{~s}$ & 2308713055 & $548 \mathrm{~s}$ & 82944 & $<1 s$ \\
\hline
\end{tabular}

(total deviation $4+4+1+7+14+10+7+19+5=71$ ) were solved with the state-of-the-art scores.

\section{B. Solomon-Potwin-Bengio Benchmark}

Table III shows that the solution qualities of our implementation $\left(C_{4}\right)$ wrt. the results of [20] $\left(C_{1}\right)$ and [5] $\left(C_{2}\right.$ and $C_{3}$ ). The solution qualities align with the best-known values (e.g., reported at http://iridia.ulb.ac.be/ manuel/files/ TSPTW/SolomonPotvinBengio.best). With our algorithm only one state-of-the-art solution (marked with an asterisk) has not been found in the allocated time period. We also give approximate timing information and the level $l$ (in brackets) to which the search was initialized. Aligning with the precursor work, every experiment has been repeated 2-4 times in case no state-of-the-art solution has been established.

To measure the savings for algorithm engineering we compared the number of evaluations in a complete (level 5 10-iteration) search (beam search option disabled) for the largest problem in the benchmark suite (rc204.1). The original implementation took $2 \mathrm{~m} 31 \mathrm{~s}$, while the refined implementation required only $11 \mathrm{~s}$. This documents that we have improved the mere running time of the source by for more than one order of magnitude!

We conducted one extra experiment (the only one for which extra information was included into the system). Seeding expert knowledge on the sequence of the first 5 or 10 cities visited by the salesman, helped solving the remaining problem ( $<1 \mathrm{~m}$ for a prefix of 10 cities and $<5 \mathrm{~m}$ for a prefix of 5 cities). For this case, we simply set $P_{0}(u, v)=100$ if $v$ is
TABLE II

RESULTS IN LARGER INSTANCES OF THE DUMAS TSPTW BENCHMARK (COSTS OF THE OBTAINEd SOlutions $C_{m}$ AND CPU TIMES $T_{m}$ ARE SHOWN, COST REFER TO THE BEST KNOWN SOLUTIONS).

\begin{tabular}{|c|c|c|c|c|c|c|}
\hline Problem & Cost & $C_{m}$ & Problem & Cost & $C_{m}$ & $T_{m}$ \\
\hline $\mathrm{n} 40 \mathrm{w} 20.001$ & 500 & $500<15 m$ & n60w20.001 & 551 & 551 & $<15 m$ \\
\hline n40w20.002 & 552 & $552<15 m$ & n60w20.002 & 605 & 605 & $<15 m$ \\
\hline n40w20.003 & 478 & $478<15 m$ & n60w20.003 & 533 & 533 & $<15 m$ \\
\hline n40w20.004 & 404 & $404<15 m$ & n60w20.004 & 616 & 616 & $<15 m$ \\
\hline n40w20.005 & 499 & $499<15 m$ & n60w20.005 & 603 & 603 & $<15 m$ \\
\hline n40w40.001 & 465 & $465<15 m$ & n60w40.001* & 591 & 595 & $=15 \mathrm{~m}$ \\
\hline $\mathrm{n} 40 \mathrm{w} 40.002$ & 461 & $461<15 m$ & n60w40.002 & 621 & 621 & $<15 m$ \\
\hline $\mathrm{n} 40 \mathrm{w} 40.003$ & 474 & $474<15 m$ & n60w40.003 & 603 & 603 & $<15 m$ \\
\hline n40w40.004 & 452 & $452<15 m$ & n60w40.004 & 597 & 597 & $<15 m$ \\
\hline $\mathrm{n} 40 \mathrm{w} 40.005$ & 453 & $453<15 m$ & $\mathrm{n} 60 \mathrm{w} 40.005$ & 539 & 539 & $<15 m$ \\
\hline n40w60.001 & 494 & $494<15 m$ & n60w60.001 & 609 & 609 & $<15 m$ \\
\hline n40w60.002 & 470 & $470<15 m$ & n60w60.002* & 566 & 571 & $=15 \mathrm{~m}$ \\
\hline n40w60.003* & 408 & $412=15 \mathrm{~m}$ & n60w60.003* & 485 & 486 & $=15 \mathrm{~m}$ \\
\hline n40w60.004 & 382 & $382<15 m$ & n60w60.004 & 571 & 571 & $<15 m$ \\
\hline n40w60.005 & 328 & $328<15 m$ & n60w60.005* & 569 & 576 & $=15 \mathrm{~m}$ \\
\hline n40w80.001 & 395 & $395<15 m$ & n60w80.001 & 458 & 458 & $<15 m$ \\
\hline n40w80.002* & 431 & $435=15 \mathrm{~m}$ & n60w80.002 & 498 & 498 & $<15 m$ \\
\hline n40w80.003 & 412 & $412<15 m$ & n60w80.003 & 550 & 550 & $<15 m$ \\
\hline n40w80.004 & 417 & $417<15 m$ & n60w80.004* & 566 & 580 & $=15 \mathrm{~m}$ \\
\hline n40w80.005 & 344 & $344<15 m$ & n60w80.005* & 468 & 478 & $=15 \mathrm{~m}$ \\
\hline n40w100.001* & 429 & $438=15 \mathrm{~m}$ & n60w100.001 & 515 & 515 & $<15 m$ \\
\hline n40w100.002 & 358 & $358<15 m$ & n60w100.002* & 538 & 545 & $=15 \mathrm{~m}$ \\
\hline n40w100.003 & 364 & $364<15 m$ & n60w100.003* & 560 & 589 & $=15 \mathrm{~m}$ \\
\hline n40w100.004 & 357 & $357<15 m$ & n60w100.004 & 510 & 510 & $<15 m$ \\
\hline n40w100.005 & 377 & $377<15 m$ & n60w100.005* & 451 & 556 & $=15 \mathrm{~m}$ \\
\hline
\end{tabular}

a successor of $u$ in the optimal solution. More complex nonautomated hints provided by domain experts are possible.

\section{Solomon-Peasant Benchmark}

In Table IV we consider the benchmark by Solomon and Peasant, for which we have not found a recent publication to compare with. We provide solution cost results, the number of rollouts performed, and the CPU time of a Level-8 search at or close to the moment, when the best solution is found or when it hits the time threshold of $15 \mathrm{~m}$. In contrast to the Solomon-Potwin-Bengio benchmark, we document results of one straight run of the algorithm, which solves all but 4 of the 27 problem instances with the state-of-the-art cost value.

\section{AFG Benchmark}

In Table $\mathrm{V}$ we show the AFG benchmark results. We provide explorations results of a Level-8 search at (or close to) the moment, when the best solution was found, or when it hit the time threshold of $15 \mathrm{~m}$. Again we document results of one straight run of the algorithm, which solves all of the simpler problems $(N<40)$ with the state-of-the-art scores, but not the harder problems in the set (results for $N>160$ are skipped).

\section{E. Langevin Benchmark}

The Langevin benchmark is a larger set of problems that appears to be simpler for mTSP. As documented in Table VI the entire set of $N=20$ problems is solved matching the state-of-the-art cost in $1 \mathrm{~s}$, the entire set of $N=40$ benchmark problems is presumably optimally solved in less than $10 \mathrm{~s}$, and in one single $15 \mathrm{~m}$ runs only three problems are not resulting in the state-of-the-art, cost leaving only a small margin to improve. 
TABLE III

RESUlts In SOLOMON-Potwin-Bengio TSPTW BENCHMARK (COST REFERS TO THE BEST KNOWN SOLUTION, $C_{1}$ IS THE COST COMPUTED BY NMC+DOMAIN-DEPENDENT PRUNING RULES, $C_{2}$ THE COST COMPUTED BY NPRA, AND $C_{3}$ THE COST COMPUTED BY

NPRA+DOMAIN-DEPENDENT EXTENSIONS; $C_{m}\left(T_{m}\right)$ THE COST (CPU TIME) COMPUTED BY MTSP).

\begin{tabular}{|l|r|r|rrr|rr|}
\hline Problem & $N$ & Cost & $C_{1}$ & $C_{2}$ & $C_{3}$ & $C_{m}$ & $T_{m}$ \\
\hline rc206.1 & 4 & 117.85 & 117.85 & 117.85 & 117.85 & $117.85(5)$ & $<1 m$ \\
rc207.4 & 6 & 119.64 & 119.64 & 119.64 & 119.64 & $119.64(5)$ & $<1 m$ \\
rc202.2 & 14 & 304.14 & 304.14 & 304.14 & 304.14 & $304.14(5)$ & $<1 m$ \\
rc205.1 & 14 & 343.21 & 343.21 & 343.21 & 343.21 & $343.21(5)$ & $<1 m$ \\
rc203.4 & 15 & 314.29 & 314.29 & 314.29 & 314.29 & $314.29(5)$ & $<1 m$ \\
rc203.1 & 19 & 453.48 & 453.48 & 453.48 & 453.48 & $453.48(5)$ & $<1 m$ \\
rc201.1 & 20 & 444.54 & 444.54 & 444.54 & 444.54 & $444.54(5)$ & $<1 m$ \\
rc204.3 & 24 & 455.03 & 455.03 & 455.03 & 455.03 & $455.03(5)$ & $<1 m$ \\
rc206.3 & 25 & 574.42 & 574.42 & 574.42 & 574.42 & $574.42(5)$ & $<1 m$ \\
rc201.2 & 26 & 711.54 & 711.54 & 711.54 & 711.54 & $711.54(5)$ & $<30 m$ \\
rc201.4 & 26 & 793.64 & 793.64 & 793.64 & 793.64 & $793.64(5)$ & $<30 m$ \\
rc205.2 & 27 & 755.93 & 755.93 & 755.93 & 755.93 & $755.93(5)$ & $<30 m$ \\
rc202.4 & 28 & 793.03 & 793.03 & 800.18 & 793.03 & $793.03(5)$ & $<30 m$ \\
rc205.4 & 28 & 760.47 & 760.47 & 765.38 & 760.47 & $760.47(5)$ & $<2 h$ \\
rc202.3 & 29 & 837.72 & 837.72 & 839.58 & 839.58 & $837.72(5)$ & $<2 h$ \\
rc208.2 & 29 & 533.78 & 536.04 & 537.74 & 533.78 & $533.78(5)$ & $<2 h$ \\
rc207.2 & 31 & 701.25 & 707.74 & 702.17 & 701.25 & $701.25(8)$ & $<2 h$ \\
rc201.3 & 32 & 790.61 & 790.61 & 796.98 & 790.61 & $790.61(5)$ & $<30 m$ \\
rc204.2 & 33 & 662.16 & 675.33 & 673.89 & 664.38 & $662.16(8)$ & $<30 m$ \\
rc202.1 & 33 & 771.78 & 776.47 & 775.59 & 772.18 & $771.78(8)$ & $<30 m$ \\
rc203.2 & 33 & 784.16 & 784.16 & 784.16 & 784.16 & $784.16(5)$ & $<30 m$ \\
rc207.3 & 33 & 682.40 & 687.58 & 688.50 & 682.40 & $682.40(5)$ & $<2 h$ \\
rc207.1 & 34 & 732.68 & 743.29 & 743.72 & 738.74 & $732.68(5)$ & $<2 h$ \\
rc205.3 & 35 & 825.06 & 828.27 & 828.36 & 825.06 & $825.06(5)$ & $<30 m$ \\
rc208.3 & 36 & 634.44 & 641.17 & 656.40 & 650.49 & $634.44(8)$ & $<2 h$ \\
rc203.3 & 37 & 817.53 & 837.72 & 820.93 & 817.53 & $817.53(5)$ & $<2 h$ \\
rc206.2 & 37 & 828.06 & 839.18 & 829.07 & 828.06 & $828.06(8)$ & $<2 h$ \\
rc206.4 & 38 & 831.67 & 859.07 & 831.72 & 831.67 & $831.67(5)$ & $<30 m$ \\
rc208.1* & 38 & 789.25 & 797.89 & 799.24 & 793.60 & $793.60(8)$ & $<2 h$ \\
rc204.1 & 46 & 868.76 & 899.79 & 883.85 & 880.89 & $878.64(8)$ & $<2 h$ \\
\hline
\end{tabular}

\section{CONCLUSION AND OUTLOOK}

Nested Monte-Carlo (NMC) search is a more recent randomized single-agent state space search techniques that has proven to quickly find good solutions to a growing number of combinatorial problems with huge state spaces and large branching factors.

We have seen that knowledge and algorithm engineering can greatly improve NMC search for solving the TSPTW problems. Algorithm engineering for the existing code leads to an improvement of more than factor 10 in the exploration efficiency, whereas knowledge engineering is included to seed the policy for finding good solutions early. The results are promising and a challenge to state-of-the-art TSPTW solvers, including evolutionary algorithms and ant colony optimization. We have matched most, but not improved any best-known solution from the repository (which we assume are all optimal).

Our long-term goal is to improve our application scenario: industrial vehicle routing for improved logistics in a multiagent simulation system. Here, the agents solve individual TSP problems and trade their solution for improving the overall cost. The TSP are generated by shortest path reduction of a map wrt. to pickup and delivery locations of costumers as well as the depot(s) and vehicle fleed of the distributor. Besides time window constraints we are confronted with a variety of
TABLE IV

RESUlTS IN SOlOMON-PEASANT TSPTW BENCHMARK (COST IS THE BEST KNOWN SOLUTION; $C_{m}, E_{m}$ AND $T_{m}$ ARE THE PERFORMANCE INDICATORS COMPUTED BY MTSP).

\begin{tabular}{|c|c|c|c|c|c|}
\hline Problem & $N$ & Cost & $C_{m}$ & $E_{m}$ & $T_{m}$ \\
\hline $\mathrm{rc} 201.0$ & 26 & 628.62 & 628.62 & 40000 & $<1 s$ \\
\hline $\mathrm{rc} 201.1$ & 29 & 654.70 & 654.70 & 50000 & $<1 s$ \\
\hline $\mathrm{rc} 201.2$ & 29 & 707.65 & 707.65 & 10000 & $<1 s$ \\
\hline $\mathrm{rc} 201.3$ & 20 & 422.54 & 422.54 & 10000 & $<1 s$ \\
\hline $\mathrm{rc} 202.0$ & 26 & 496.22 & 496.22 & 120000 & $<1 s$ \\
\hline rc202.1 & 23 & 426.53 & 426.53 & 60000 & $<1 s$ \\
\hline $\mathrm{rc} 202.2$ & 28 & 611.77 & 611.77 & 20000 & $<1 s$ \\
\hline rc202.3 & 27 & 627.85 & 627.85 & 130000 & $<2 s$ \\
\hline $\mathrm{rc} 203.0$ & 36 & 727.45 & 727.45 & 3000000 & $<3 m$ \\
\hline $\mathrm{rc} 203.1$ & 38 & 726.99 & 726.99 & 1500000 & $<3 m$ \\
\hline $\mathrm{rc} 203.2$ & 29 & 617.46 & 617.46 & 20000 & $<20 s$ \\
\hline $\mathrm{rc} 204.0$ & 33 & 541.45 & 541.45 & 11100000 & $<10 m$ \\
\hline rc204.1 & 29 & 485.37 & 485.37 & 20000000 & $<14 m$ \\
\hline $\mathrm{rc} 204.2$ & 41 & 778.40 & 778.40 & 4150000 & $<6 m$ \\
\hline $\mathrm{rc} 205.0$ & 27 & 511.65 & 511.65 & 80000 & $<5 s$ \\
\hline $\mathrm{rc} 205.1$ & 23 & 491.22 & 491.22 & 20000 & $<1 s$ \\
\hline $\mathrm{rc} 205.2^{*}$ & 29 & 714.69 & 717.56 & 35310000 & $=15 \mathrm{~m}$ \\
\hline $\mathrm{rc} 205.3$ & 25 & 601.24 & 601.24 & 90000 & $<5 s$ \\
\hline $\mathrm{rc} 206.0$ & 36 & 835.23 & 835.23 & 1500000 & $<1 m$ \\
\hline $\mathrm{rc} 206.1$ & 34 & 664.73 & 664.73 & 1140000 & $<40 s$ \\
\hline $\mathrm{rc} 206.2^{*}$ & 33 & 655.37 & 670.80 & 33110000 & $=15 \mathrm{~m}$ \\
\hline rc207.0 & 38 & 806.69 & 806.69 & 6000000 & $<4 m$ \\
\hline $\mathrm{rc} 207.1$ & 34 & 726.36 & 726.36 & 20000000 & $<11 m$ \\
\hline $\mathrm{rc} 207.2$ & 31 & 546.41 & 546.41 & 3600000 & $<2 m$ \\
\hline $\mathrm{rc} 208.0^{*}$ & 45 & 820.56 & 884.96 & 14220000 & $=15 \mathrm{~m}$ \\
\hline $\mathrm{rc} 208.1^{*}$ & 28 & 509.04 & 558.48 & 35210000 & $=15 \mathrm{~m}$ \\
\hline $\mathrm{rc} 208.2$ & 30 & 503.92 & 503.92 & 7700000 & $<4 m$ \\
\hline
\end{tabular}

additional side-constraints: limited driving times and requested breaks for the driver premium contracts, pickup and backhault tasks, just to name a few. We would use the real world orders, implement a large agent system with a sufficient amount of agents, cluster all orders of the day staticly for each cluster we solve the TSP with the solver, and for each cluster we compute a lower bound (e.g., with the Hungrian algorithm) As result, we solve different TSPs based on a real infrastructure, have real properties of orders, and could determine the quality of the results by the distance to the optimal solution. 
TABLE VI

RESULTS IN LANGEVIN TSPTW BENCHMARK.

TABLE V

RESULTS IN AFG TSPTW BENCHMARK

\begin{tabular}{|c|c|c|c|c|c|}
\hline Problem & $N$ & Cost & $C_{m}$ & $E_{m}$ & $T_{m}$ \\
\hline rbg010a & 11 & 671 & 671 & 50000 & $<1 s$ \\
\hline rbg016a & 17 & 938 & 939 & 50000 & $<1 s$ \\
\hline rbg016b & 17 & 1304 & 1304 & 100000 & $<5 s$ \\
\hline $\operatorname{rbg} 017.2$ & 18 & 852 & 852 & 50000 & $<1 s$ \\
\hline rbg017 & 16 & 893 & 893 & 50000 & $<1 s$ \\
\hline rbg017a & 18 & 4296 & 4296 & 50000 & $<1 s$ \\
\hline rbg019a & 20 & 1262 & 1262 & 50000 & $<1 s$ \\
\hline $\operatorname{rbg} 019 b$ & 20 & 1866 & 1866 & 50000 & $<1 s$ \\
\hline $\operatorname{rbg} 019 \mathrm{c}$ & 20 & 4536 & 4545 & 2566080 & $=15 \mathrm{~m}$ \\
\hline rbg019d & 20 & 1356 & 1356 & 50000 & $<1 s$ \\
\hline rbg020a & 21 & 4689 & 4689 & 50000 & $<1 s$ \\
\hline $\operatorname{rbg} 021.2$ & 22 & 4528 & 4528 & 100000 & $<5 s$ \\
\hline $\operatorname{rbg} 021.3$ & 22 & 4528 & 4528 & 150000 & $<5 s$ \\
\hline $\operatorname{rbg} 021.4$ & 22 & 4525 & 4525 & 200000 & $<10 \mathrm{~s}$ \\
\hline $\operatorname{rbg} 021.5$ & 22 & 4515 & 4515 & 2000000 & $<1 m$ \\
\hline $\operatorname{rbg} 021.6$ & 22 & 4480 & 4480 & 150000 & $<5 s$ \\
\hline $\operatorname{rbg} 021.7$ & 22 & 4479 & 4479 & 100000 & $<5 s$ \\
\hline rbg021.8 & 22 & 4478 & 4478 & 150000 & $<5 s$ \\
\hline rbg021.9 & 22 & 4478 & 4478 & 200000 & $<10 s$ \\
\hline rbg021 & 22 & 4536 & 4536 & 50000 & $<1 s$ \\
\hline rbg027a & 28 & 5091 & 5051 & 1000000 & $<30 s$ \\
\hline rbg031a & 32 & 1863 & 1863 & 200000 & $<10 \mathrm{~s}$ \\
\hline rbg033a & 34 & 2069 & 2069 & 400000 & $<20 s$ \\
\hline $\operatorname{rbg} 034 a$ & 35 & 2222 & 2222 & 500000 & $<30 s$ \\
\hline g035a.2 & 36 & 2056 & 2056 & 7558272 & $<15 \mathrm{~m}$ \\
\hline g035a & 36 & 2144 & 2144 & 1000000 & $<30 s$ \\
\hline & 39 & 480 & 2480 & 000 & $<30 s$ \\
\hline rbg040a* & 41 & 2378 & 2413 & 18242496 & $=15 \mathrm{~m}$ \\
\hline rbg041a* & 42 & 2598 & 2625 & 16842816 & $=15 \mathrm{~m}$ \\
\hline rbg042a* & 43 & 2772 & 2805 & 15723072 & $=15 \mathrm{~m}$ \\
\hline rbg048a* & 49 & 9383 & 9480 & 398080 & $=15 \mathrm{~m}$ \\
\hline rbg049a* & 50 & 10018 & 10075 & 9097020 & $=15 \mathrm{~m}$ \\
\hline $\operatorname{rbg} 050 a^{*}$ & 51 & 2953 & 974 & 6625152 & $=15 \mathrm{~m}$ \\
\hline $\operatorname{rbg} 050 b^{*}$ & 51 & 9863 & 9921 & 8724672 & $=15 \mathrm{~m}$ \\
\hline $\operatorname{rbg} 050 c^{*}$ & 51 & 10024 & 10088 & 6998400 & $=15 \mathrm{~m}$ \\
\hline rbg055a & 56 & 3761 & 3761 & 10000 & $<10 s$ \\
\hline rbg067a & 68 & 4625 & 4625 & 4000000 & $<5 m$ \\
\hline rbg086a* & 87 & 8400 & 8418 & 3405888 & $=15 \mathrm{~m}$ \\
\hline rbg092a* & 93 & 7160 & 7197 & 2846016 & $=15 \mathrm{~m}$ \\
\hline $\operatorname{rbg} 125 \mathrm{a}^{*}$ & 125 & 7936 & 8012 & 1772928 & $=15 \mathrm{~m}$ \\
\hline $\operatorname{rbg} 132.2^{*}$ & 133 & 8200 & 8489 & 1259712 & $=15 \mathrm{~m}$ \\
\hline & 133 & 8470 & 8668 & 1259712 & $=15 \mathrm{~m}$ \\
\hline $\operatorname{rbg} 152.3^{*}$ & 153 & 9797 & 10571 & 793152 & $=15 \mathrm{~m}$ \\
\hline $\operatorname{rbg} 152 *$ & 153 & 10032 & 10229 & 793152 & $=15 \mathrm{~m}$ \\
\hline
\end{tabular}

\begin{tabular}{|c|c|c|c|c|}
\hline Problem & $N$ & Cost & $C_{m}$ & $T_{m}$ \\
\hline N20ft301 & 20 & 61.60 & 661.60 & \\
\hline $20 \mathrm{ft} 302$ & 0 & & & \\
\hline & & & & \\
\hline & & & & \\
\hline & & & & \\
\hline $\mathrm{N} 20 \mathrm{ft}$ & 20 & 727.80 & & \\
\hline & & & & \\
\hline & 20 & & & \\
\hline & & & & \\
\hline & 20 & & & \\
\hline 01 & 20 & & & \\
\hline & & & & \\
\hline 93 & 20 & & & \\
\hline & & & & \\
\hline 05 & 20 & & & \\
\hline & & 727 & & \\
\hline & 20 & & & $<$ \\
\hline & & & & \\
\hline & 20 & & & \\
\hline & 20 & & & \\
\hline & 40 & & & $1 \mathrm{~m}$ \\
\hline 2 & 40 & & & $<1$ \\
\hline & 40 & & & \\
\hline & 40 & & & $<1 n$ \\
\hline & 40 & & & \\
\hline & 40 & & & $<1 m$ \\
\hline & 40 & & & \\
\hline & 40 & & & $<1 m$ \\
\hline & 40 & & & $<1$ \\
\hline & 40 & & & \\
\hline & 40 & & & $<1 m$ \\
\hline & 40 & & & \\
\hline & 40 & & & $<1 m$ \\
\hline & & & & \\
\hline & 40 & & & $<1 m$ \\
\hline & & & & \\
\hline & 40 & & & $<1 m$ \\
\hline & 40 & & & $<1 m$ \\
\hline & 40 & & & \\
\hline & 40 & & & $<1 m$ \\
\hline & 60 & & & $<15 m$ \\
\hline & & & & $<15 m$ \\
\hline & & & & $<15 m$ \\
\hline & 60 & & & $15 \mathrm{~m}$ \\
\hline & & & & $<15$ \\
\hline & 60 & & & $<15 m$ \\
\hline & & & & $<15 m$ \\
\hline & 60 & & & $<15 m$ \\
\hline & & & & $<15 m$ \\
\hline & 60 & & & $<15 m$ \\
\hline & & & & $<15 m$ \\
\hline & 60 & & & $<15 m$ \\
\hline & & & & $<15 m$ \\
\hline & 60 & & & $<15 m$ \\
\hline & 60 & & & $<15 m$ \\
\hline & 60 & & & $<15 m$ \\
\hline & 60 & & & $<15 m$ \\
\hline & 60 & & & $<15 m$ \\
\hline & 60 & & & $<15 m$ \\
\hline & 60 & & & $<15 m$ \\
\hline & 60 & & & $<15 m$ \\
\hline & 60 & & & $=15 \mathrm{~m}$ \\
\hline & 60 & & & $<15 m$ \\
\hline & 60 & & & $<15 m$ \\
\hline & 60 & & & $<15 m$ \\
\hline & 60 & & & $=15 \mathrm{~m}$ \\
\hline & 60 & & & $=15 \mathrm{~m}$ \\
\hline & 60 & & & $<15 m$ \\
\hline & 60 & & & $<15 m$ \\
\hline N60ft410 & 60 & 1189.60 & 1189.60 & $<15 m$ \\
\hline
\end{tabular}




\section{REFERENCES}

[1] P. Auer, N. Cesa-Bianchi, and P. Fischer. Finite-time analysis of the multiarmed bandit problem. Machine Learning, 47(2-3):235-256, 2002.

[2] E. Baker. An exact algorithm for the time-constrained traveling salesman problem. Operations Research, 31(5):938-945, 1983.

[3] T. Cazenave. Monte-Carlo Beam Search. IEEE Transactions on Computational Intelligence and AI in Games 4(1): 68-72, 2012.

[4] T. Cazenave. Nested Monte-Carlo search. In IJCAI, pages 456-461, 2009.

[5] T. Cazenave and F. Teytaud. Application of the nested rollout policy adaptation algorithm to the traveling salesman problem with time windows. In LION, pages 42-54, 2011.

[6] N. Christofides, A. Mingozzi, and P. Toth. State-space relaxation procedures for the computation of bounds to routing problems. Networks, 11(2):145-164, 1981.

[7] Y. Dumas, J. Desrosiers, E. Gelinas, and M. Solomon. An optimal algorithm for the traveling salesman problem with time windows. Operations Research, 43(2):367-371, 1995.

[8] S. Edelkamp and M. Gath. Optimal decision making in agent-based autonomous groupage traffic. In ICAART, 2013. To appear.

[9] H. Finnsson and Y. Björnsson. Simulation-based approach to general game playing. In $A A A I$, pages 1134-1139, 2008.

[10] F. Focacci, A. Lodi, and M. Milano. A hybrid exact algorithm for the TSPTW. INFORMS Journal on Computing, 14(4):403-417, 2002.

[11] S. Gelly and Y. Wang. Exploration exploitation in Go: UCT for MonteCarlo Go. In NIPS-Workshop on On-line Trading of Exploration and Exploitation, 2006.

[12] M. Gendreau, A. Hertz, G. Laporte, and M. Stan. A generalized insertion heuristic for the traveling salesman problem with time windows. Operations Research, 46(3):330-335, 1998.

[13] R. Jonker and A. Volgenant. Improving the hungarian assignment algorithm. Operations Research Letters, 5:171-175, 1986.

[14] L. Kocsis and C. Szepesvari. Bandit based Monte-Carlo planning. In ICML, pages 282-293, 2006

[15] M. Lopez-Ibanez and C. Blum. Beam-ACO for the travelling salesman problem with time windows. Computers \& OR, 37(9):1570-1583, 2010.

[16] N. C. Love, T. L. Hinrichs, and M. R. Genesereth. General game playing: Game description language specification. Technical Report LG-2006-01, Stanford Logic Group, 2006

[17] S. Parragh, K. Doerner, and Richard Hartl. A survey on pickup and delivery problems. Journal für Betriebswirtschaft, 58(2):81-117, 2008.

[18] G. Pesant, M. Gendreau, J. Potvin, and J. Rousseau. An exact constraint logic programming algorithm for the traveling salesman problem with time windows. Transportation Science, 32(1):12-29, 1998.

[19] J. Potvin and S. Bengio. The vehicle routing problem with time windows part II: genetic search. INFORMS Journal on Computing, 8(2):165, 1996.

[20] A. Rimmel, F. Teytaud, and T. Cazenave. Optimization of the nested Monte-Carlo algorithm on the traveling salesman problem with time windows. In Applications of Evolutionary Computation, pages 501-510, 2011.

[21] A. Rimmel, F. Teytaud, and O. Teytaud. Biasing Monte-Carlo simulations through rave values. In Computers and Games, pages 59-68, 2011.

[22] C. D. Rosin. Nested rollout policy adaptation for Monte-Carlo tree search. In IJCAI, pages 649-654, 2011.

[23] M. Solomon. Algorithms for the vehicle routing and scheduling problems with time window constraints. Operations Research, 35(2):254265, 1987. 